\title{
A case of an 81 -year-old with cough and dyspnea
}

\begin{abstract}
It is uncommon to diagnose usual interstitial pneumonitis as a unilateral presentation. We present a case of an 81-year-old current smoker who presented with exertional dyspnea and dry cough. The patient had right sided UIP pattern in the CT chest along with hiatus hernia. The etiology for the unilateral lung involvement was postulated to be due to the hiatus hernia leading to gastro-esophageal reflux disease (GERD) which caused micro aspirations leading to lung injury and fibroblast activation. Whether this can be prevented by anti-reflux medications needs further research. Our patient was managed with pirfenidone, metered dose inhalers containing tiotropium and proton-pump inhibitors. Thus, a high index of suspicion for underlying gastro-esophageal reflux must be kept in such patients to arrive at an early diagnosis and start treatment.
\end{abstract}

Key words: unilateral UIP, GERD, hiatus hernia

Adv Respir Med. 2020; 88: 454-457

\section{Introduction}

Usual interstitial pneumonitis (UIP) pattern is often seen in idiopathic pulmonary fibrosis, rheumatoid arthritis associated interstitial lung disease, chronic hypersensitivity pneumonitis, asbestosis along with progressive fibrosing interstitial lung diseases. The pattern is described with bilateral subpleural basal reticular opacities with honeycombing, with or without traction bronchiectasis. Occasionally, there can be a unilateral presentation of this pattern leading to diagnostic dilemma. We present a case of an elderly male patient who had an unilateral UIP with an interesting underlying probable etiology.

\section{Case report}

An 81-year-old man, current smoker with 30 pack-years smoking history, presented to the out-patient department with complaints of gradually progressive exertional dyspnea for 4 months associated with dry cough, more in the evening. There was no history of fever, hemoptysis, loss of weight or appetite. The patient was on proton-pump inhibitors (PPI) irregularly since last 6 years for gastroesophageal reflux disease (GERD). There were no other comorbidities. He denied history of joint pains, Raynaud phenomenon, skin tightening, dry eyes, dry mouth or any skin rash. The patient had no other drug intake, relevant family history and has negative autoimmune markers.

Examination of the chest revealed right sided end-inspiratory Velcro-like crackles. Arterial blood gas analysis revealed the following parameters: $\mathrm{pH}-7.41, \mathrm{pO}_{2}-63, \mathrm{pCO}_{2}-39$ and bicarbonate $\left(\mathrm{HCO}_{3}\right)-24$. A spirometry was suggestive of FEV1/FVC (forced expiratory volume in one second/forced vital capacity) of 63 , FEV1 $-40 \%$ and FVC - 65\%; signifying obstruction with possible restriction, which was confirmed by a low total lung capacity. The chest X-ray and chest CT scans are attached (Figures 1-5).

The case was discussed in multidisciplinary discussion and both the radiologist and pulmonologist opined that it fulfils the criteria of usual interstitial pneumonia pattern. The etiology of unilateral involvement was postulated as gastroesophageal reflux disease (GERD) with recurrent aspirations in view of the nocturnal cough, use of PPI and a large hiatus hernia evident on CT

Address for correspondence: Pranav Ish, Vardhman Mahavir Medical College \& Safdarjung Hospital, New Delhi, India; e-mail: pranavish2512@gmail.com

DOI: 10.5603/ARM.a2020.0158

Received: 20.02.2020

Copyright (C) 2020 PTChP

ISSN 2451-4934 
scan. A study done by Raghu et al. has shown that GERD prevalence is up to $87 \%$ in idiopathic pulmonary fibrosis (IPF) with only half of them having symptoms [1]. Another study suggested that nearly $66 \%$ of patients with IPF had reflux and symptoms did not help to diagnose it [2]. Hence, in the absence of symptoms, diagnosing GERD early and identifying it as a risk factor for initiation and progression of IPF is difficult. However, GERD associated with recurrent aspirations, preferably on the right side can lead to lung injury and fibroblast activation which may be responsible for unilateral IPF. Whether this can be prevented by anti-reflux medica-

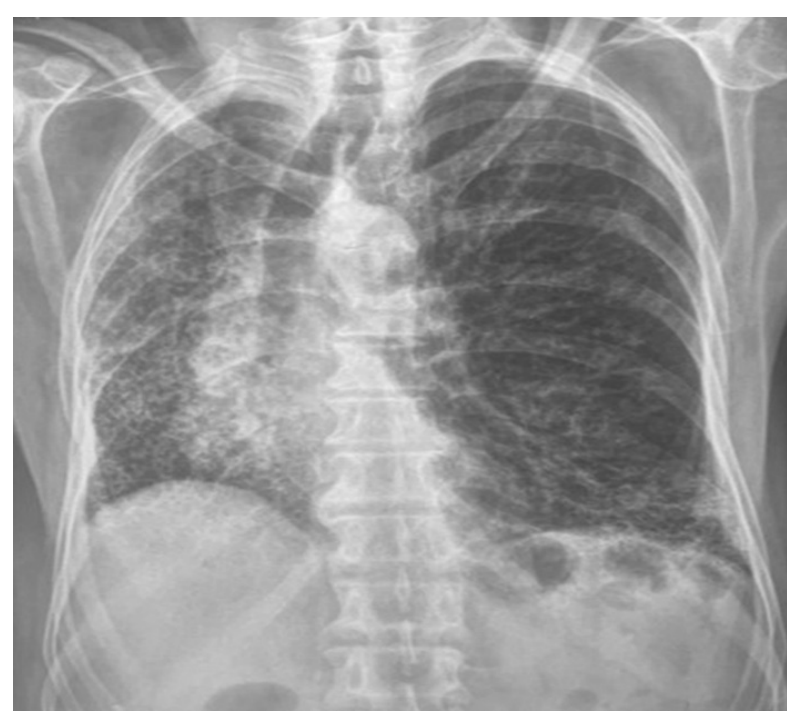

Figure 1. Frontal chest radiograph showing volume loss in the right hemithorax with ipsilateral cardio-mediastinal shift. Diffuse reticular opacities with preferential involvement of the right side and an apicobasal gradient on the left side tions needs further research. Our patient was discharged on pirfenidone, metered dose inhalers containing tiotropium and proton-pump inhibitors. He did not give consent to surgical lung biopsy.

Thus, a final diagnosis of unilateral usual interstitial pneumonitis (UIP) with underlying hiatus hernia causing GERD was made. In view of negative drug history, autoimmune history and investigations, occupational or exposure history, a diagnosis of unilateral IPF was considered.

\section{Discussion}

The literature on unilateral IPF and its plausible mechanisms is sparse [2]. A study of 14 asymmetrical IPF when compared to symmetrical IPF revealed similar clinical characteristics, including more frequent occurrence in men, elderly population with history of smoking with a trend towards increased mortality over an eight-year follow-up. The etiology of the unilateral nature of the disease could not be ascertained as GERD, prior radiation therapy to the chest and smoking history was not found to be significantly different. The authors, however, admitted to the small sample size to be able to find any causative etiologies [3].

Another large study of asymmetrical IPF investigated 96 patients, out of which 32 had asymmetrical IPF, but none had unilateral disease. This study found GERD and micro-aspirations as a plausible mechanism of the asymmetrical disease, which was supported by the increased right lung involvement [4]. A case of a middle-aged man with unilateral UIP revealed associated pulmonary artery sarcoma which can be the un-
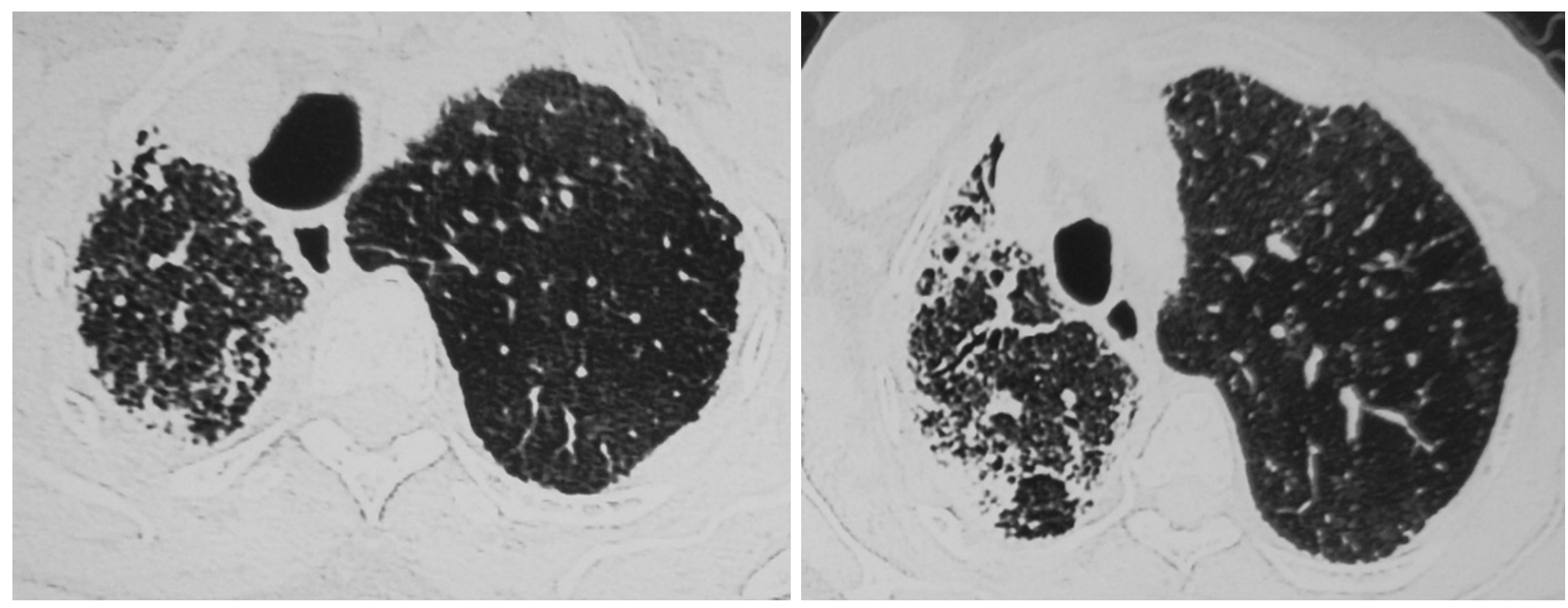

Figure 2. Axial sections through the upper lung zone show relative sparing of the lung apices and preferential involvement of the right side with compensatory hyperinflation on the left side. There is mild peripheral subpleural reticulations and traction bronchiolectasis 


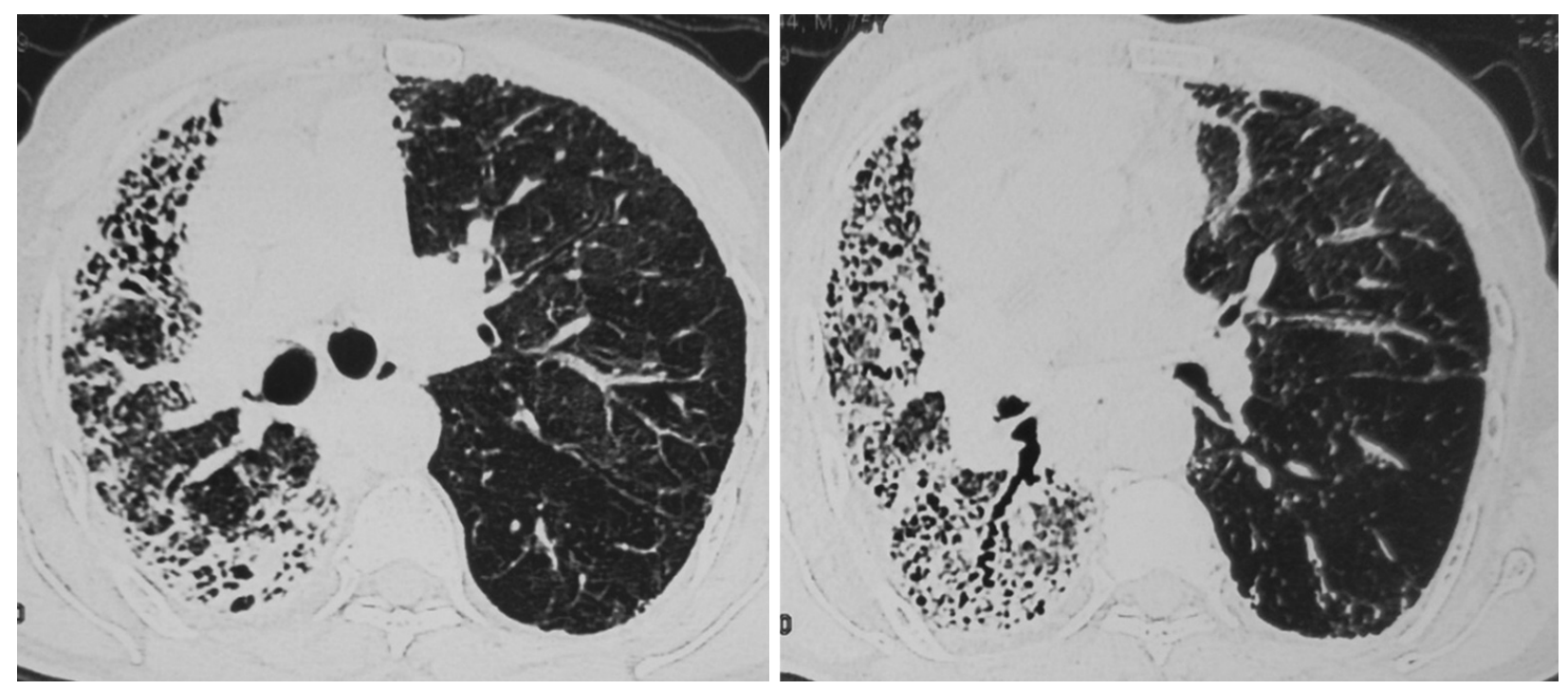

Figure 3. Axial sections through the carina and bronchus intermedius show apicobasal gradient with prominent traction bronchiectasis, fibrotic opacities, interlobular septal thickening on the right side with associated volume loss

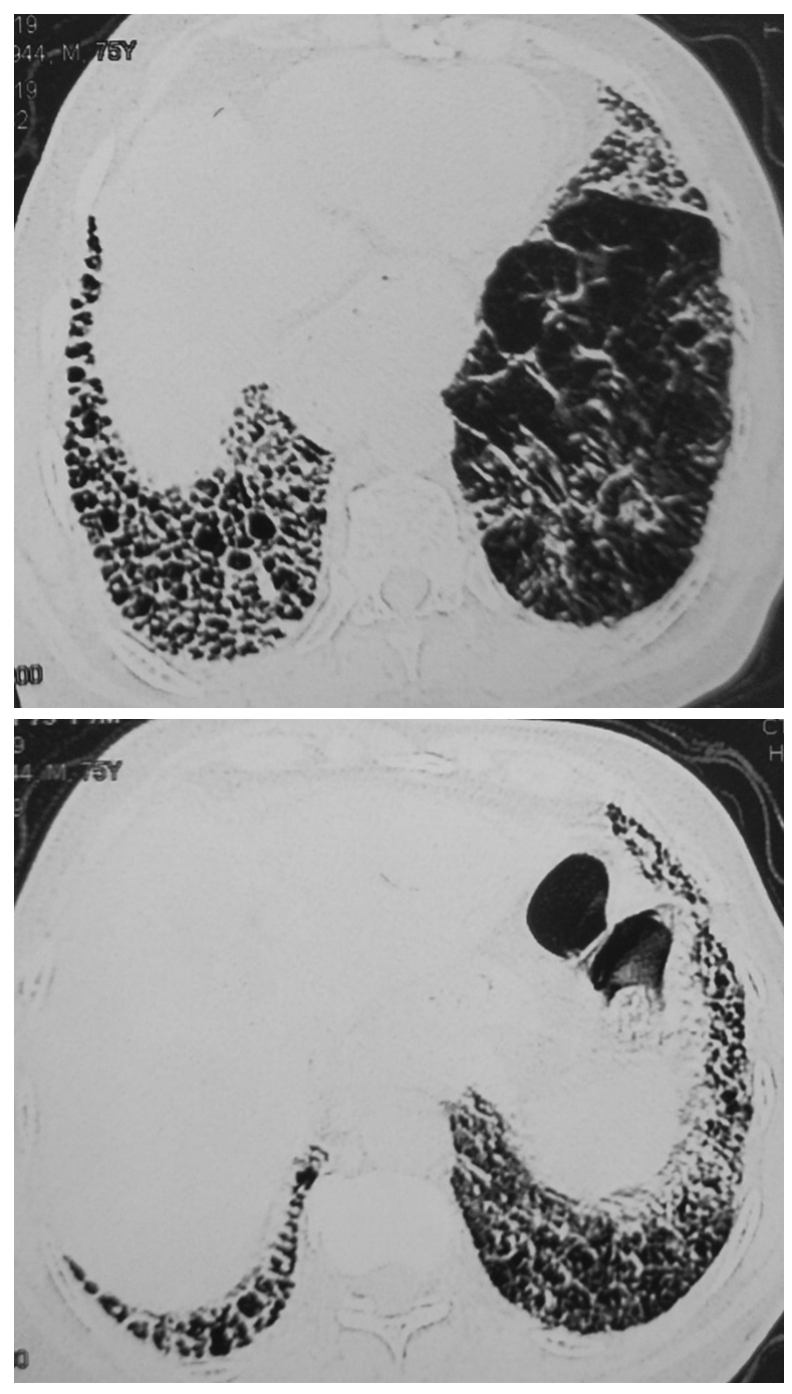

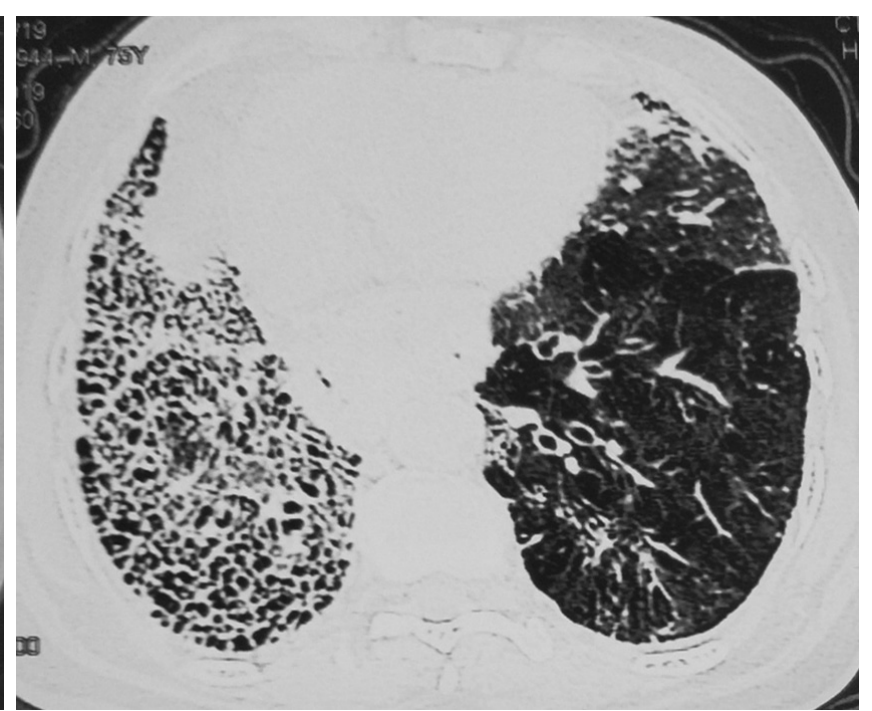

Figure 4. Axial sections through the lower lobes show diffuse traction bronchiectasis and peripheral subpleural honeycombing on the right side. The left lower lobe reveals mild peripheral reticulations and interlobular septal thickening with underlying air trapping and bronchiectasis 


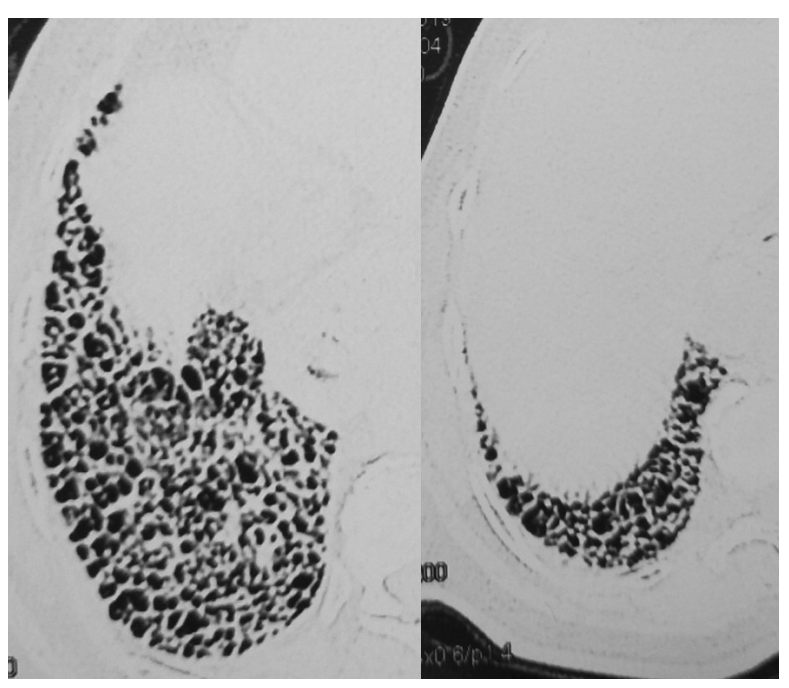

Figure 5. Magnified images of the right lower hemithorax showing peripheral subpleural honeycombing with basal predominance

derlying etiology in view of chronic pulmonary ischemia or the tumor producing some fibrogenic factors [5].

Two case reports of unilateral UIP were reported to be associated with connective tissue disorders - a young female with systemic sclerosis had GERD as the possible etiology of unilateral right sided UIP and a 70-year-old female with Sjogren syndrome with unilateral UIP who presented with an exacerbation [6, 7]. There is an interesting case report of Sweyer James Macleod syndrome (SJMS) presenting with unilateral UIP. The authors postulated that SJMS depletes the lung of source of myofibroblast which include epithelial cells and myofibroblasts. This leads to a protective effect for that lung, and eventually, the patient develops unilateral UIP only in the contralateral side [8].

\section{Conclusion}

To conclude, the existing literature on unilateral UIP is limited to case reports and case series, in view of its uncommon occurrence. However, a high index of suspicion must be kept for underlying possible causes like GERD resulting from hiatus hernia in such patients.

\section{Conflict of interest}

None declared.

\section{References:}

1. Raghu G, Freudenberger TD, Yang S, et al. High prevalence of abnormal acid gastro-oesophageal reflux in idiopathic pulmonary fibrosis. Eur Respir J. 2006; 27(1): 136-142, doi: 10.1183/09031936.06.00037005, indexed in Pubmed: 16387946.

2. Sweet MP, Patti MG, Leard LE, et al. Gastroesophageal reflux in patients with idiopathic pulmonary fibrosis referred for lung transplantation. J Thorac Cardiovasc Surg. 2007; 133(4): 1078-1084, doi: 10.1016/j.jtcrs.2006.09.085, indexed in $\mathrm{Pu}-$ bmed: 17382656 .

3. Callahan SJ, Xia M, Murray S, et al. Clinical characteristics in patients with asymmetric idiopathic pulmonary fibrosis. Respir Med. 2016; 119: 96-101, doi: 10.1016/j.rmed.2016.08.028, indexed in Pubmed: 27692155.

4. Tcherakian C, Cottin V, Brillet PY, et al. Progression of idiopathic pulmonary fibrosis: lessons from asymmetrical disease. Thorax. 2011; 66(3): 226-231, doi: 10.1136/thx.2010.137190, indexed in Pubmed: 20880868.

5. Chong S, Kim TS, Chung MP, et al. Unilateral usual interstitial pneumonia associated with sarcoma of the pulmonary artery. AJR Am J Roentgenol. 2007; 189(4): W221-W223, doi: 10.2214/ AJR.05.1011, indexed in Pubmed: 17885035.

6. Lesnyak VN, Danilevskaya OV, Averyanov AV, et al. Unilateral pulmonary fibrosis and systemic sclerosis. Am J Respir Crit Care Med. 2014; 190(9): 1067-1068, doi: 10.1164/rccm.201407-1253IM, indexed in Pubmed: 25360729.

7. Takahashi T, Satoh M, Satoh H. Unilateral acute exacerbation of pulmonary fibrosis in association with Sjögren's syndrome. Intern Med. 1996; 35(10): 811-814, doi: 10.2169/internalmedicine.35.811, indexed in Pubmed: 8933193.

8. Abdulla O, Cain J, Howells J. Swyer-James-MacLeod syndrome with unilateral pulmonary fibrosis: a case report. BJR Case Rep. 2017; 3(4): 20160105, doi: 10.1259/bjrcr.20160105, indexed in Pubmed: $\underline{30363238}$. 